\title{
ОДНОВРЕМЕННОЕ ВЛИЯНИЕ ВРАЩЕНИЯ ОБРАЗЦА И ВЧ ПОЛЕЙ НА СПЕКТРЫ ЯМР ТВЕРДЫХ ТЕЛ: ВОЗМОЖНОСТЬ СЕЛЕКТИВНОГО НАБЛЮДЕНИЯ ВЗАИМОДЕЙТВИЙ
}

Механическое вращение образца обусловливает, как известно, гармоническую зависимость от времени пространственных координат в гамильтониане, описывающем эволюцию спин-системы. Благодаря этому в случае достаточно большой частоты $\omega_{r}$ и определенным образом выбранной оси вращения все анизотропные взаимодействия усредняются до нуля, и спектр ЯМР твердых тел определяется гамильтонианом, аналогичным гамильтониану жидкостей $\left.{ }^{1}\right]$. Спектр, полученный в соответствующем эксперименте, состоит из узких линий и не содержит информации об анизотропных взаимодействиях.

С другой стороны, известно, что воздействие на образец ВЧ полей обусловливает гармоническую зависимость от времени спиновых координат в спин-гамильтониане. И в этом случае есть возможность с помощью соответствующим образом выбранного непрерывного ВЧ поля $\left[{ }^{2,3}\right]$ или последовательностью ВЧ импульсов $\left[{ }^{4}\right]$ получить в спектрах твердых тел узкие линии, что указывает на усреднение некоторых взаимодействий.

Весьма существен тот факт, что временная зависимость отдельных членов гамильтониана, на основе которой проводится их усреднение, вводится в этих двух случаях по-разному. Именно поэтому одновременное использование вращения и ВЧ полей при определенном выборе их параметров позволяет устранить зависимость от времени тех членов гамильтониана, которые в случае применения только вращения или только ВЧ полей оказывались зависящими от времени. Так 'как выражения гамильтонианов различных взаимодействий в общем случае различны, то можно надеяться, что условия, накладываемые на параметры вращения и ВЧ полей, селективны относительно взаимодействий. Это означает, что одновременное использование вращения и ВЧ полей даст возможность селективно сохранять (восстанавливать) влияние на эволюцию спин-системы того или иного типа взаимодействия. Тем самым предлагаемый метод открывает принципиальную возможность селективного изучения взаимодействий. Ниже выясняются условия, при которых тот или иной тип взаимодействия эффективно влияет на эволюцию спин-системы. 


\section{О гамильтониане, эффективно управляющем состоянием спин-системы}

1. Описание образца и эксперимента. Рассмотрим монокристалл, спин-система которого содержит большое число I-спинов и малое число $\mathrm{S}$-спинов. Начиная с момента $t=0$ образец вращается с частотой $\omega_{r}$ в сильном постоянном магнитном поле $(z-$ направление лабораторных координат (ЛК)). Затем в момент $t=t_{1}$ включаются два ВЧ поля, направленные по оси $x$ ЛК и влияющие непосредственно на I- и S-спины:

$$
\vec{H}(t)=\vec{i}\left[2 H^{I} \cos \omega_{1}\left(t-t_{1}\right)+2 H^{s} \cos \omega_{2}\left(t-t_{1}\right)\right], \quad t>t_{1} .
$$

Ось вращения расположена в плоскости $y z$ ЛК и образует с осью $z$ угол $\Theta_{m}$.

Предполагается, что состояние спин-системы @ подчиняется уравнению Лиувилля

$$
\dot{\varrho}=-\mathrm{i} \Omega(\mathbf{H}) \mathrm{Q},
$$

где

$$
\Re(H) Q=[H, Q] .
$$

Гамильтониан в уравнении (2) является суммой членов, описывающих взаимодействия в спиновой системе:

$$
\mathbf{H}=\mathbf{H}_{z}^{I}+\mathbf{H}_{c}^{I}+\mathbf{H}_{d}^{I I}+\mathbf{H}_{J}^{I I}+\mathbf{H}_{z}^{S}+\mathbf{H}_{c}^{S}+\mathbf{H}_{d}^{I S}+\mathbf{H}_{J}^{I S}+\mathbf{H}_{r f}^{I}+\mathbf{H}_{r f}^{S} .
$$

Учитывая низкую концентрацию S-спинов, всеми взаимодействиями между ними можно пренебречь.

Отдельные члены гамильтониана (4) выбираются в следующем виде:

$$
\begin{aligned}
& \mathbf{H}_{z}^{I}=-\sum_{i} \omega^{I} \mathbf{I}_{z i}, \\
& \mathbf{H}_{c}^{I}=\sum_{i} \omega^{I} \sum_{k=x, y, z} \sigma_{i k z}^{L I} \mathbf{I}_{k i}, \\
& \mathbf{H}_{d}^{I I}=\sum_{i<j} C_{i j}\left(1-3 \cos ^{2} \Theta_{i j}^{L}\right)\left(\mathbf{I}_{z i} \mathbf{I}_{z j}-\frac{1}{3} \overrightarrow{\mathbf{I}}_{i} \overrightarrow{\mathbf{I}}_{j}\right), \\
& \mathbf{H}_{J}^{I I}=\sum_{i<j} J_{i j} \overrightarrow{\mathbf{I}}_{i} \overrightarrow{\mathbf{I}}_{j}, \\
& \mathbf{H}_{z}^{S^{*}}=-\omega^{S} \mathbf{S}_{z}, \\
& \mathbf{H}_{c}^{S}=\omega^{S} \sum_{k=x, y, z} \sigma_{k z}^{L S} \mathbf{S}_{k}, \\
& \mathbf{H}_{d}^{I S}=\mathbf{S}_{z} \sum_{n} C_{n}\left(1-3 \cos ^{2} \Theta_{n}^{L}\right) \mathbf{I}_{z n}, \\
& \mathbf{H}_{J}^{I S}=\mathbf{S}_{z} \sum_{n} J_{n} \mathbf{I}_{z n},
\end{aligned}
$$




$$
\begin{aligned}
& \mathbf{H}_{r f}^{I}=-2 \sum_{i} h^{I} \mathbf{I}_{x i} \cos \omega_{1} t, \\
& \mathbf{H}_{r f}^{S}=-2 h^{S} S_{x} \cos \omega_{2} t, \\
& C_{(i j)(n)}=\frac{3 \gamma_{I} \gamma_{(I)(S)} h}{2 r_{(i j)(n)}^{3}},
\end{aligned}
$$

содержание остальных обозначений традиционное. Индексом $L$ снабжены величины, относящиеся к ЛК (константы спин-спиновых связей $J_{i j}$ и $J_{n}$ предполагаются изотропными). Кроме того, в выражениях (11) и (12) учтены взаимодействия S-спина только с ближайшими I-спинами.

C целью нахождения гамильтониана, эффективно управляющего состоянием спин-системы в период воздействия как вращения, так и ВЧ полей, за начало отсчета времени в выражениях (5)-(14) принят момент $t_{1}$, а текущее время обозначено по-прежнему символом $t$.

Отметим, что вследствие вращения образца члены гамильтониана $\mathbf{H}_{c}^{I}, \mathbf{H}_{d}^{I I}, \mathbf{H}_{\mathrm{c}}^{S}$ и $\mathbf{H}_{d}^{I S}$ также изменяются во времени. Выражая содержащиеся в них величины $\alpha^{L}$ через соответствующие величины $\alpha$ в системе координат, связанной с образцом (СКСО), получим, что эти члены содержат независящие от времени слагаемые и слагаемые, зависящие от времени с частотами $\omega_{r}$ и $2 \omega_{r}$. В качестве СКСО в данной работе принята вращающаяся вместе с образцом система координат, ось $z$ которой совпадает с осью вращения, а ось $x-$ с осью $x$ ЛК в начальный момент вращения.

2. Об эффективном га мильтони ане. Учет при решении уравнения (2) всех зависящих от времени членов в гамильтониане (4), с одной стороны, мягко говоря, затруднителен, а с другой, по крайней мере при определенных условиях эксперимента, - и необязателен. Чтобы уточнить существенные, с точки зрения эволюции состояния спин-системы, члены гамильтониана, преобразуем входящие в уравнение (2) величины и проведем приближение.

Во-первых, предпримем следующее преобразование

$$
\mathbf{Q}_{R}=\mathbf{R}^{-1} \mathbf{Q R}^{+1} ; \quad \mathbf{R}^{ \pm 1}=\exp \left[ \pm \mathrm{i} t_{\omega_{1}} \sum_{i} \mathbf{I}_{z i} \pm \mathrm{i} t \omega_{2} \mathbf{S}_{z}\right],
$$

что можно трактовать как переход от ЛК к системе координат, вращающихся вокруг оси $z$ с частотами ВЧ полей. В результате для определения $\varrho_{R}(t)$ получим уравнение, совпадающее по виду с уравнением (2).

Во-вторых, переведем уравнение для $\varrho_{R}(t)$ в представление, которое можно интерпретировать как поворот $z$-оси вращающейся системы координат вдоль эффективного поля в ней. Это есть преобразование

$$
\mathbf{Q}_{T}=\mathbf{T}^{+1} \mathbf{Q}_{R} \mathbf{T}^{-1} ; \quad \mathbf{T}^{ \pm 1}=\exp \left[ \pm \mathrm{i} \Theta^{I} \sum_{i} \mathbf{I}_{y i} \pm \mathrm{i} \Theta^{S} \mathbf{S}_{y}\right],
$$

где

$$
\begin{gathered}
\cos \Theta^{(I)(S)}=\frac{\Delta^{(I)(S)}}{h_{e f}^{(I)(S)}}, \\
\Delta^{(I)(S)}=\omega^{(I)(S)}-\omega_{(1)(2)} \\
h_{e f}^{(I)(S)}=\sqrt{\left(\Delta^{(I)(S)}\right)^{2}+\left(h^{(I)(S)}\right)^{2}} .
\end{gathered}
$$


Наконец, применим к величинам в полученном уравнении преобразование

$$
\mathbf{Q}_{v}=\mathbf{V}^{-1} \mathbf{Q}_{T} \mathbf{V}^{+1} ; \quad \mathbf{V}^{ \pm 1}=\exp \left[ \pm \mathrm{i} t h_{e f}^{I} \sum_{i} \mathbf{I}_{z i} \pm \mathrm{i} t h_{e f}^{\mathrm{S}} \mathbf{S}_{z}\right],
$$

что можно рассматривать как вращение вокруг наклоненной во вращающейся системе координат $z$-оси. Теперь уравнение Лиувилля принимает вид

$$
\dot{\mathrm{Q}}_{v}=-\mathrm{i} \Omega(\tilde{\mathbf{H}}) \varrho v,
$$

где

$$
\tilde{\mathbf{H}}=\mathbf{H}_{c v}^{I I}+\mathbf{H}_{d v}^{I I}+\underset{J v}{\mathbf{H}^{I I}}+\mathbf{H}_{c v} \mathbf{H}_{d v}+\mathbf{H}_{J v}^{I S}+\underset{r f, v}{\mathbf{H}^{I S}}+\mathbf{H}_{r f, v}^{I, 2}+\mathbf{H}_{r, 2}^{S, 2},
$$

a $\mathbf{H}_{r f, v}^{I, 2}$ и $\mathbf{H}_{r f, v}^{S, 2}$ содержат только те части описывающих влияние ВЧ полей гамильтонианов, которые после преобразования (16) остались зависимыми от времени (с частотами $2 \omega_{1}$ и $2 \omega_{2}$ соответственно).

Уравнение (21) с гамильтонианом (22) является точным в том смысле, что мы получили его из формулы (2) только посредством вышеперечисленных преобразований, не прибегая к приближениям.

Для дальнейшего изложения предположим, что все частоты достаточно велики

$$
\left|\omega_{1}\right|,\left|\omega_{2}\right| \gg\left|\omega_{r}\right|,\left|h_{e f}^{I}\right|,\left|h_{e f}^{S}\right| \gg\left|h_{L}\right|,
$$

где $h_{L}$ - величина т. н. локальных полей, и рассмотрим только те варианты эксперимента, при которых существенной в уравнении (21) является лишь независящая от времени часть $\mathbf{H}_{e f}$ гамильтониана $\tilde{\mathbf{H}}$ (22). В этом случае в качестве решения уравнения (21) имеем

$$
\varrho_{v}(t)=\mathrm{e}^{-i t \mathfrak{Q}\left(\mathbf{H}_{e}\right)} \varrho_{0 v},
$$

и регистрируемый в эксперименте спектр отражает эффективный гамильтониан $\mathbf{H}_{e f}$. Оператор @ov описывает состояние спин-системы в момент $t_{1}$.

Анализ показывает, что компоненты $\tilde{\mathbf{H}}$ могут содержать два независящих от времени типа слагаемых: 1) которые постоянны во времени вне зависимости от конкретных значений $\omega_{r}, h_{e f}^{I}, h_{e f}^{S}$ и 2) которые ста. новятся постоянными во времени и, следовательно, входят в $\mathbf{H}_{e f}$ при определенных значениях $\omega_{r}, h_{e f}^{I}, h_{e f}^{S}$. Выражения всех возможных постоянных слагаемых приведены в Приложении. Обозначение взаимодействия снабжено выражением «const» в случае первого типа слагаемых и условием, при выполнении которого данное слагаемое оказывается постоянным, в случае второго типа.

Существенно различные варианты экспериментальных условий, при которых $\mathbf{H}_{e f}$ содержит постоянные слагаемые второго типа (ПСВТ), представлены в таблице. При одновременном выполнении нескольких условий из таблицы $\mathbf{H}_{e f}$ имеет столько ПСВТ, сколько их в соответствующих столбцах. Если же не выполняется ни одно из условий, $\mathbf{H}_{e f}$ содержит лишь независящие от времени слагаемые первого типа.

Из выражений постоянных слагаемых (см. Приложение) видно, что эффективный гамильтониан $\mathbf{H}_{e f}$ имеет нанболее простой вид в случае точной настройки ВЧ полей, $\Delta^{I}=\Delta^{S}=0$, при оси вращения, образующей с осью $z$ ЛК магический угол $\cos ^{2} \Theta_{m}=1 / 3$. Тогда все постоянные слагаемые первого типа, кроме спин-спиновой связи между I-спинами, 


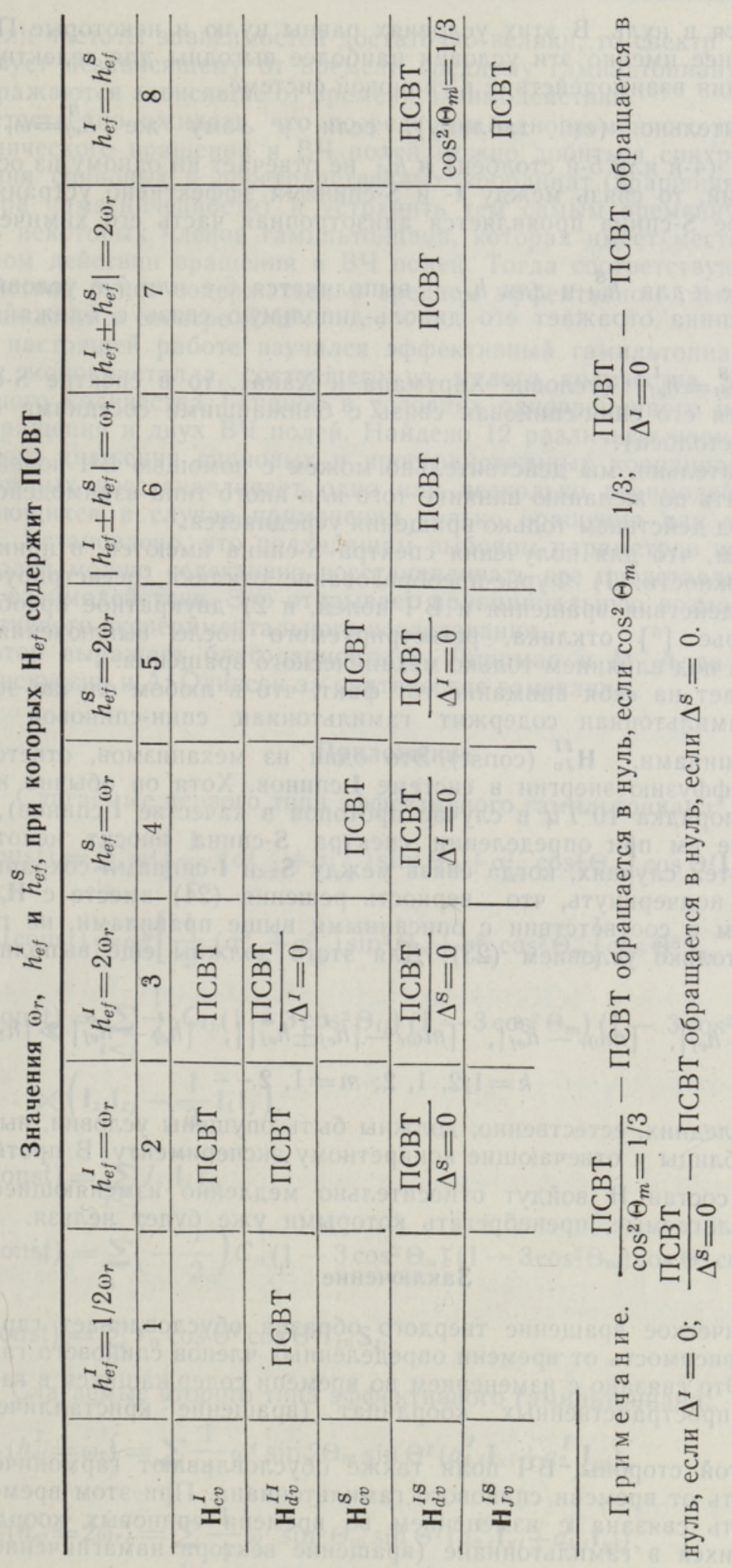


обращаются в нуль. В этих условиях равны нулю и некоторые ПСВТ. Тем не менее именно эти условия нанболее выгодны для селективного исследования взаимодействий в спиновой системе.

Действительно (см. таблицу), если к тому же $h_{e j}^{\mathrm{S}}=\omega_{r}$ или $h_{e f}^{S}=2 \omega_{r} \quad$ (4-й или 5 -й столбец) и $h_{e f}^{I}$ не отвечает ни одному из остальных условий, то связь между I- и S-спинами эффективно устраняется, a в спектре $\mathrm{S}$-спина проявляется анизотропная часть его химического сдвига.

Если же и для $h_{e f}^{S}$ и для $h_{e f}^{I} \quad$ выполняется 6-е или 7 -е условие, то спектр S-спина отражает его диполь-дипольную связь с ближайшими I-спинами.

Если $h_{e f}^{S}=h_{e f}^{I} \quad$ (условие Хартмана и Хана), то в спектре S-спина проявляется его спин-спиновая связь с ближайшими соседними I-спинами (8-й столбец).

Следовательно, мы действительно можем с помощью ВЧ полей восстанавливать по желанию влияние того или иного типа взаимодействия, которое под действием только вращения усредняется.

Отметим, что для получения спектра S-спина имеются, в принципе, две возможности: 1) Фурье-преобразование отклика, регистрируемого во время действия вращения и ВЧ полей, и 2) двукратное преобразование Фурье $\left[{ }^{5}\right]$ отклика, регистрируемого после выключения ВЧ полей, т. е. под влиянием только механического вращения.

Обращает на себя внимание тот факт, что в любом случае эффективный гамильтониан содержит гамильтониан спин-спиновой связи между I-спинами, $\mathbf{H}_{J v}^{I I}$ (const). Это один из механизмов, ответственных за диффузию энергии в системе I-спинов. Хотя он обычно незначителен (порядка $10 \Gamma \iota$ в случае протонов в качестве I-спинов), пренебрежение им при определении спектра S-спина вносит некоторую ошибку в тех случаях, когда связь между S- и I-спинами сохраняется.

Важно подчеркнуть, что верность решения (24) вместе с $\mathbf{H}_{e f}$, составленным в соответствии с описанными выше правилами, не гарантируется только условием (23). Для этого должны еще выполняться условия

$$
\begin{gathered}
\left|k_{\omega_{r}}-h_{e f}^{I}\right|, \quad\left|m_{\omega_{r}}-h_{e f}^{S}\right|, \quad\left|m_{\omega_{r}}-\right| h_{e f}^{I} \pm h_{e f}^{S}||, \quad\left|h_{e f}^{I}-h_{e f}^{S}\right| \gg\left|h_{L}\right|, \\
k=1 / 2,1,2 ; m=1,2 .
\end{gathered}
$$

Среди последних, естественно, должны быть опущены условия, выбранные из таблищы и отвечающие конкретному эксперименту. В противном случае в состав $\tilde{\mathbf{H}}$ войдут относительно медленно изменяющиеся во времени слагаемые, пренебрегать которыми уже будет нельзя.

\section{Заключение}

Механическое вращение твердого образца обусловливает гармоническую зависимость от времени определенных членов спинового гамильтониана. Это связано с изменением во времени содержащихся в гамильтониане пространственных координат (вращение кристаллического каркаса).

С другой стороны, ВЧ поля также обусловливают гармоническую зависимость от времени спинового гамильтониана. При этом временная зависимость связана с изменением во времени спиновых координат, содержащихся в гамильтониане (вращение вектора намагниченности). 
Если частоты зависимостей достаточно велики, то спектр ЯМР соответствует независящему от времени среднему гамильтониану, и в нем не отражаются зависящие от времени взаимодействия.

Естественно ожидать, что посредством одновременного применения механического вращения и ВЧ полей можно добиться синхронного изменения спиновых и пространственных координат (вращения каркаса и вектора намагниченности) и устранить тем самым временную зависимость некоторых членов гамильтониана, которая имеет место при раздельном действии вращения и ВЧ полей. Тогда соответствующее взаимодействие будет содержаться в среднем эффективном гамильтониане и отражаться в спектре ЯМР.

В настоящей работе изучался эффективный гамильтониан спин-системы монокристалла, состоящего из малого количества S-спинов и большого количества I-спинов в условиях одновременного использования вращения и двух ВЧ полей. Найдено 12 различных вариантов синхронного движения спиновых и пространственных координат, каждый из которых восстанавливает одно или несколько взаимодействий, усредняющихся в случае применения только вращения или только ВЧ полей. Установлено, что подходящим выбором параметров вращения и ВЧ полей можно селективно восстанавливать все представляющие интерес взаимодействия. Это открывает принципиальную возможность их селективного экспериментального исследования.

Автор выражает благодарность Э. Липпмаа и М. Алла за полезные дискуссии и А. Оливсон за критические замечания.

\section{Приложение}

А. Слагаемые первого типа эффективного гамильтониана:

$$
\begin{aligned}
& \mathbf{H}_{c v}^{I} \text { (const) }=\sum_{i} \omega^{I}\left[\frac{1}{2}\left(\sigma_{i x x}^{I}+\sigma_{i y y}^{I}\right) \sin ^{2} \Theta_{m}+\sigma_{i z z}^{I} \cos ^{2} \Theta_{m}\right] \cos \Theta^{I} \mathbf{I}_{z i}, \\
& \mathbf{H}_{c v}^{S}=(\text { const })=\omega^{S}\left[\frac{1}{2}\left(\sigma_{x x}^{S}+\sigma_{y y}^{S}\right) \sin ^{2} \Theta_{m}+\sigma_{z z}^{S} \cos ^{2} \Theta_{m}\right] \cos \Theta^{S} \mathbf{S}_{z}, \\
& \mathbf{H}_{d v}^{I I} \text { (const) }=\sum_{i<j} \frac{1}{4} C_{i j}\left(1-3 \cos ^{2} \Theta_{i j}\right)\left(1-3 \cos ^{2} \Theta_{m}\right)\left(1-3 \cos ^{2} \Theta^{I}\right) \times \\
& \times\left(\mathbf{I}_{z i} \mathbf{I}_{z j}-\frac{1}{3} \overrightarrow{\mathbf{I}}_{i} \overrightarrow{\mathbf{I}}_{j}\right),
\end{aligned}
$$

Б. Слагаемые второго типа эффективного гамильтониана:

1. $\mathbf{H}_{\mathrm{cv}}^{I}\left(h_{e f}^{I}=\omega_{r}\right)=\sum_{i} \frac{1}{4} \omega^{I} \sin 2 \Theta_{m} \sin \Theta^{I}\left(a_{1, i}^{I} \mathbf{I}_{x i}+a_{2, i}^{I} \mathbf{I}_{y i}\right)$,
$\mathbf{H}_{c v}^{I}\left(h_{e j}^{I}=2 \omega_{r}\right)=\sum_{i} \frac{1}{4} \omega^{I} \sin ^{2} \Theta_{m} \sin \Theta^{I}\left(a_{3, i}^{I} \mathbf{I}_{x i}+a_{4, i}^{I} \mathbf{I}_{y i}\right)$, 
где

$$
\begin{aligned}
& a_{1, i}^{I}=\left(\sigma_{i y z}^{I}+\sigma_{i z y}^{I}\right) \cos \omega_{r} t_{1}+\left(\sigma_{i x z}^{I}+\sigma_{i z x}^{I}\right) \sin \omega_{r} t_{1}, \\
& a_{2, i}^{I}=\left(\sigma_{i x z}^{I}+\sigma_{i z x}^{I}\right) \cos \omega_{r} t_{1}-\left(\sigma_{i y z}^{I}+\sigma_{i z y}^{I}\right) \sin \omega_{r} t_{1}, \\
& a_{3, i}^{I}=\left(\sigma_{i x x}^{I}-\sigma_{i y y}^{I}\right) \cos 2 \omega_{r} t_{1}-\left(\sigma_{i x y}^{I}+\sigma_{i y x}^{I}\right) \sin 2 \omega_{r} t_{1}, \\
& a_{4, i}^{I}=-\left[\left(\sigma_{i x y}^{I}+\sigma_{i y x}^{I}\right) \cos 2 \omega_{r} t_{1}+\left(\sigma_{i x x}^{I}-\sigma_{i y y}^{I}\right) \sin 2 \omega_{r} t_{1}\right] .
\end{aligned}
$$

2. $\mathbf{H}_{\mathrm{cv}}^{\mathrm{S}}\left(h_{e f}^{\mathrm{S}}=\omega_{r}\right)=\frac{1}{4} \omega^{\mathrm{S}} \sin 2 \Theta_{m} \sin \Theta^{\mathrm{S}}\left(a_{1}^{\mathrm{S}} \mathbf{S}_{x}+a_{2}^{\mathrm{S}} \mathbf{S}_{y}\right)$

$$
\mathbf{H}_{\mathrm{cv}}^{\mathrm{S}}\left(h_{e f}^{\mathrm{S}}=2 \omega_{r}\right)=\frac{1}{4} \omega^{S} \sin ^{2} \Theta_{m} \sin \Theta^{S}\left(a_{3}^{S} \mathbf{S}_{x}+a_{4}^{S} \mathbf{S}_{y}\right),
$$

a величины $a_{k}^{S}$ получаются из $a_{h, i}^{I}$ заменой в последних $\sigma_{i}^{I}$ на $\sigma^{S}$.

3. $\mathbf{H}_{d v}^{I I}\left(h_{e f}^{I}=\frac{1}{2} \omega_{r}\right)=\sum_{i<j} \frac{3}{4} C_{i j} \cos \Theta_{i j} \sin 2 \Theta_{m} \sin ^{2} \Theta^{I} \times$

$$
\begin{aligned}
& \times\left[b_{1, i j}\left(\mathbf{I}_{x i} \mathbf{I}_{x j}-\mathbf{I}_{y i} \mathbf{I}_{y j}\right)+b_{2, i j}\left(\mathbf{I}_{x i} \mathbf{I}_{y j}+\mathbf{I}_{y i} \mathbf{I}_{x j}\right)\right], \\
\mathbf{H}_{d v}^{I I}\left(h_{e f}^{I}=\omega_{r}\right)= & \sum_{i<j}\left(-\frac{3}{4}\right) C_{i j} \cos \Theta_{i j} \sin 2 \Theta_{m} \sin 2 \Theta^{I} \times \\
& \times\left[b_{1, i j}\left(\mathbf{I}_{z i} \mathbf{I}_{x j}+\mathbf{I}_{x i} \mathbf{I}_{z j}\right)+b_{2, i j}\left(\mathbf{I}_{z i} \mathbf{I}_{y j}+\mathbf{I}_{y i} \mathbf{I}_{z j}\right)\right]+ \\
& +\sum_{i<j}\left(-\frac{3}{8}\right) C_{i j} \sin ^{2} \Theta_{m} \sin ^{2} \Theta^{I} \times \\
& \times\left[b_{3, i j}\left(\mathbf{I}_{x i} \mathbf{I}_{x j}-\mathbf{I}_{y i} \mathbf{I}_{y j}\right)+b_{4, i j}\left(\mathbf{I}_{x i} \mathbf{I}_{y j}+\mathbf{I}_{y i} \mathbf{I}_{x j}\right)\right], \\
\mathbf{H}_{d v}^{I I}\left(h_{e f}^{I}=2 \omega_{r}\right)= & \sum_{i<j} \frac{3}{8} C_{i j} \sin ^{2} \Theta_{m} \sin 2 \Theta^{I} \times \\
& \times\left[b_{3, i j}\left(\mathbf{I}_{z i} \mathbf{I}_{x j}+\mathbf{I}_{x i} \mathbf{I}_{z j}\right)+b_{4, i j}\left(\mathbf{I}_{z i} \mathbf{I}_{y j}+\mathbf{I}_{y i} \mathbf{I}_{z j}\right)\right],
\end{aligned}
$$

где

$b_{1, i j}=\cos \beta_{i j} \cos \omega_{r} t_{1}+\cos \alpha_{i j} \sin \omega_{r} t_{1}$,

$b_{2, i j}=\cos \alpha_{i j} \cos \omega_{r} t_{1}-\cos \beta_{i j} \sin \omega_{r} t_{1}$,

$b_{3, i j}=\left(\cos ^{2} \beta_{i j}-\cos ^{2} \alpha_{i j}\right) \cos 2 \omega_{r} t_{1}+2 \cos \alpha_{i j} \cos \beta_{i j} \sin 2 \omega_{r} t_{1}$,

$b_{4, i j}=2 \cos \alpha_{i j} \cos \beta_{i j} \cos 2 \omega_{r} t_{1}-\left(\cos ^{2} \beta_{i j}-\cos ^{2} \alpha_{i j}\right) \sin 2 \omega_{r} t_{1}$,

а $\cos \alpha_{i j}$ и $\cos \beta_{i j}-$ направляющие косинусы вектора $\vec{r}_{i j}$ относительно осей $x$ и $y$ СКСО соответственно.

4. $\mathbf{H}_{d v}^{I S}\left(h_{e f}^{I}=h_{e f}^{S}\right)=\sum_{n}\left(-\frac{1}{4}\right) C_{n}\left(1-3 \cos ^{2} \Theta_{n}\right)\left(1-3 \cos ^{2} \Theta_{m}\right) \times$

$$
X \sin \Theta^{I} \sin \Theta^{S}\left(\mathbf{I}_{x n} \mathbf{S}_{x}+\mathbf{I}_{y n} \mathbf{S}_{y}\right),
$$

$$
\begin{aligned}
\mathbf{H}_{d v}^{I S}\left(h_{e f}^{I}=\omega_{r}\right)=\sum_{n}( & \left.-\frac{3}{2}\right) C_{n} \cos \Theta_{n} \sin 2 \Theta_{m} \sin \Theta^{I} \cos \Theta^{S} \times \\
& \times\left[b_{1, n} \mathbf{I}_{x n} \mathbf{S}_{z}+b_{2, n} \mathbf{I}_{y n} \mathbf{S}_{z}\right]
\end{aligned}
$$




$$
\begin{aligned}
\mathbf{H}_{d v}^{I S}\left(h_{e f}^{S}=\omega_{r}\right) & =\sum_{n}\left(-\frac{3}{2}\right) C_{n} \cos \Theta_{n} \sin 2 \Theta_{m} \cos \Theta^{I} \sin \Theta^{S} \times \\
& \times\left[b_{1, n} \mathbf{I}_{z n} \mathbf{S}_{x}+b_{2, n} \mathbf{I}_{z n} \mathbf{S}_{y}\right],
\end{aligned}
$$

$\mathbf{H}_{d v}^{I S}\left( \pm h_{e f}^{S} \mp h_{e f}^{I}=\omega_{r}\right)=\sum_{n} \frac{3}{4} C_{n} \cos \Theta_{n} \sin 2 \Theta_{m} \sin \Theta^{I} \sin \Theta^{S} \times$

$$
X\left[b_{1, n}\left(\mathbf{I}_{x n} \mathbf{S}_{x}+\mathbf{I}_{y n} \mathbf{S}_{y}\right) \pm b_{2, n}\left(\mathbf{I}_{x n} \mathbf{S}_{y}-\mathbf{I}_{y n} \mathbf{S}_{x}\right)\right]
$$

$\mathbf{H}_{d v}^{I S}\left(h_{e f}^{I}+h_{e f}^{S}=\omega_{r}\right)=\sum_{n} \frac{3}{4} C_{n} \cos \Theta_{n} \sin 2 \Theta_{m} \sin \Theta^{I} \sin \Theta^{S} X$

$$
\times\left[b_{1, n}\left(\mathbf{I}_{x n} \mathbf{S}_{x}-\mathbf{I}_{y n} \mathbf{S}_{y}\right)+b_{2, n}\left(\mathbf{I}_{x n} \mathbf{S}_{y}+\mathbf{I}_{y n} \mathbf{S}_{x}\right)\right] \text {, }
$$

$\mathbf{H}_{d v}^{I S}\left(h_{e f}^{\mathrm{S}}=2 \omega_{r}\right)=\sum_{n} \frac{3}{4} C_{n} \sin ^{2} \Theta_{n} \sin \Theta^{I} \cos \Theta^{S} \times$

$$
\times\left[b_{3, n} \mathbf{I}_{x n} \mathbf{S}_{z}+b_{4, n} \mathbf{I}_{y n} \mathbf{S}_{z}\right],
$$

$\mathbf{H}_{d v}^{I S}\left(h_{e f}^{I}=2 \omega_{r}\right)=\sum_{n} \frac{3}{4} C_{n} \sin ^{2} \Theta_{m} \cos \Theta^{I} \sin \Theta^{S} X$

$$
\times\left[b_{3, n} \mathbf{I}_{z n} \mathbf{S}_{x}+b_{4, n} \mathbf{I}_{z n} \mathbf{S}_{y}\right] \text {, }
$$

$\mathbf{H}_{d v}^{I S}\left( \pm h_{e f}^{\mathrm{S}} \mp h_{e f}^{I}=2 \omega_{r}\right)=\sum_{n}\left(-\frac{3}{8}\right) C_{n} \sin ^{2} \Theta_{m} \sin \Theta^{I} \sin \Theta^{S} X$

$$
\times\left[b_{3, n}\left(\mathbf{I}_{x n} \mathbf{S}_{x}+\mathbf{I}_{y n} \mathbf{S}_{y}\right) \pm b_{4, n}\left(\mathbf{I}_{x n} \mathbf{S}_{y}-\mathbf{I}_{y n} \mathbf{S}_{x}\right)\right],
$$

$\mathbf{H}_{d v}^{I S}\left(h_{e f}^{I}+h_{e f}^{S}=2 \omega_{r}\right)=\sum_{n}\left(-\frac{3}{8}\right) C_{n} \sin ^{2} \Theta_{m} \sin \Theta^{I} \sin \Theta^{S} X$

$$
\times\left[b_{3, n}\left(\mathbf{I}_{x n} \mathbf{S}_{x}-\mathbf{I}_{y n} \mathbf{S}_{y}\right)+b_{4, n}\left(\mathbf{I}_{x n} \mathbf{S}_{y}+\mathbf{I}_{y n} \mathbf{S}_{x}\right)\right],
$$

а величины $b_{k, n}$ получаются из $b_{k, i j}$ заменой $\cos \alpha_{i j}$ и $\cos \beta_{i j}$ соответственно на $\cos \alpha_{n}$ и $\cos \beta_{n}$.

5. $\mathbf{H}_{J v}^{I S}\left(h_{e f}^{I}=\stackrel{S}{h_{e f}}\right)=\sum_{n} \frac{1}{2} J_{n} \sin \Theta^{I} \sin \Theta^{S}\left(\mathbf{I}_{x n} \mathbf{S}_{x}+\mathbf{I}_{y n} \mathbf{S}_{y}\right)$.

\section{ЛИТ Е Р А Т У Р А}

1. Andrew, E. R., Progress in nuclear magnetic resonance spectroscopy, 8, Pergamon Press, 1972

2. Redfield, A. G., Phys. Rev., 98, 1787 (1955).

3. Кундл а Э., Изв. АН ЭССР, Физ. Матем., 26, 395 (1977).

4. Ma ns í ield, P., Progress in nuclear magnetic resonance spectroscopy, 8, Pergamon Press, 1972.

5. A ue, W. P., B a rthold i, E., Ernst, R. R., J. Chem. Phys., 64, 2229 (1976).

Ннститут кибернетики Академии наук Эстонской ССР
Поступила в редакцию $18 /$ II 1977 


\section{E. KUNDLA}

\section{ROTATSIOONI JA RS-VÄLJADE SAMAAEGNE RAKENDAMINE TAHKE KEHA TMR-S: INTERAKTSIOONIDE SELEKTIIVSE UURIMISE VOIMALUS}

Tahke proovi spinnhamiltoniaani teatud liikmete harmooniline sõltuvus ajast tekib nii proovi mehaanilisel pöörlemisel kui ka rs-väljadega môjutamisel. Kui sagedused on küllalt suured, siis vastab TMR-spekter ajast sõltumatule efektiivsele hamiltoniaanile ja temas ei kajastu ajast sōltuvad interaktsioonid.

Käesolevas töös on uuritud palju I- ja vähe $\mathbf{S}$-spinne sisaldava spinnsüsteemi efektiivset hamiltoniaani mehaanilise pöörlemise ja kahe rs-välja samaaegse mõju korral. Selgub, et pööriemise ja rs-väljade parameetrite sobiva valikuga on võimalik efektiivses hamiltoniaanis selektiivselt säilitada kõiki neid interaktsioone, mis ainult mehaanilise pöörlemise vōi rs-väljade toimel väija keskmistuvad.

\section{E. KUNDLA}

\section{ON THE SIMULTANEOUS USE OF ROTATION AND RF FIELDS IN THE SOLID STATE NMR: POSSIBILITY FOR SELECTIVE INVESTIGATION OF INTERACTIONS}

Both mechanical rotation and rf fields produce harmonical time dependence of certain terms of solid probe spin hamiltonian. If the frequencies are large enough, the NMR spectrum is determined by an effective hamiltonian which does not contain any time dependent interactions.

In this paper the effective hamiltonian of the spin system containing abundant $\mathbf{I}$ and rare $\mathbf{S}$ spins in the case of simultaneous use of rotation and two rf fields was investigated. It appears that with suitabie choice of the rotation and $\mathrm{rf}$ field parameters it is possible selectively to re-establish the influence of all the interactions averaged out by the use of rotation (or ri fields) only. 